\title{
Personalization of Atrial Fibrillation Antiarrhythmic Drug Treatments: A Population of Models Approach
}

\author{
Alejandro Liberos ${ }^{1}$, Alfonso Bueno-Orovio ${ }^{2}$, Ismael Hernandez-Romero ${ }^{1,3}$, Miguel Rodrigo ${ }^{4}$, Maria \\ S. Guillem ${ }^{3}$, Francisco Fernandez-Aviles ${ }^{1}$, Felipe Atienza ${ }^{1}$, Blanca Rodriguez ${ }^{2}$, Andreu M. Climent ${ }^{1}$ \\ ${ }^{1}$ Hospital GU Gregorio Marañón, Cardiology Department, IiSGM, CIBERCV, Madrid, Spain. \\ ${ }^{2}$ Department of Computer Science, University of Oxford, Oxford, United Kingdom. \\ ${ }^{3}$ Rey Juan Carlos University, Fuenlabrada, Madrid, Spain. \\ ${ }^{4}$ ITACA, Universitat Politècnica de València, València, Spain.
}

\begin{abstract}
The efficacy of antiarrhythmic drugs terminating atrial fibrillation $(A F)$ is highly dependent on the specific genetic and epigenetic characteristics of each patient. Insilico simulations representing inter-subject variability may help in the identification of personalized ionic mechanisms underlying rotor dynamics.

In this work, a population of 173 electrophysiological atrial tissue models is used to investigate the factors promoting variability in the response of $A F$ dynamics to sodium $\left(I_{N a}\right)$ and calcium $\left(I_{C a L}\right)$ current block.

During basal conditions 126 of the 173 (72.8\%) models sustained stable reentries. Partial block of sodium terminated $A F$ by collision between rotors in 64 models (50.8\%) whereas partial calcium block produced $A F$ termination in 38 (30.2\%). Interestingly, 39 models which terminated by sodium block did not terminate by calcium block, whereas 13 which terminated by calcium block did not terminate with sodium block.

Cardiac tissue simulations accounting for inter-subject variability predict that $A F$ patients may require different treatments and will be an important tool in the classification of patients and personalized medicine.
\end{abstract}

\section{Introduction}

Antiarrhythmic drugs have a modest efficacy at the time of terminating Atrial Fibrillation (AF) and sustaining sinus rhythm in patients with long-standing AF [1].

One of the main explanations for this lack of success on chronic AF patients is that the modifications on AF substrate and perpetuation mechanisms due to prolonged episodes of the arrhythmia is highly dependent on the specific ionic current profile associated to the genetic and epigenetic characteristics of each patient [2], which may result in different perpetuation mechanisms from patient to patient.

Understanding the ionic mechanisms that govern the maintenance of $\mathrm{AF}$ drivers under a remodelled substrate and the potential differences between patients may allow the development of more effective antiarrhythmic drug treatments and a better classification of patients.

Pharmacological treatments have traditionally tried to prolong the action potential duration and refractory period of the cells resulting in an increase of wavelength. However, this strategy has presented a limited efficacy for $\mathrm{AF}$ termination and sinus rhythm maintenance [1]. Another strategy is focused on destabilizing rotor cores promoting its collision and the termination of $\mathrm{AF}$ [3].

The aim of this work was to evaluate by means of a population of 173 mathematical models the efficacy of different treatments focused in the partial block of depolarizing currents (i.e. $\mathrm{I}_{\mathrm{Na}}$ and $\mathrm{I}_{\mathrm{CaL}}$ ) for terminating $\mathrm{AF}$ by promoting the collision of rotors. Inter-subject variability allowed the identification of the different mechanisms associated with $\mathrm{AF}$ termination and thus the classification of patients according with the treatment with more probabilities of success.

\section{Methods}

An experimentally-calibrated population of 173 human AF models according to the biomarkers (Action Potential Amplitude, Action Potential Durations, Resting Membrane Potential, and dome potential) obtained from the experimental data recorded from 149 patients was used [2,4] (Figure 1A). The baseline model for used was the one developed by Koivumaki et al. [5], the resulting population comes from the random variation of eleven following ionic parameters from $-100 \%$ to $+200 \%$ of their original value $\left(\mathrm{g}_{\mathrm{Na}}\right.$, maximum $\mathrm{I}_{\mathrm{NaK}}, \mathrm{g}_{\mathrm{K} 1}, \mathrm{~g}_{\mathrm{CaL}}, \mathrm{k}_{\mathrm{NCX}}$, $\mathrm{g}_{\mathrm{to}}, \mathrm{g}_{\mathrm{Kur}}, \mathrm{g}_{\mathrm{Kr}}, \mathrm{g}_{\mathrm{Ks}}$ as well as the expression of cpumps in SERCA and the availability of $\mathrm{Ca}^{2+}$ release channels from sarcoplasmic reticulum $\mathrm{J}_{\text {rel, RyR }}$ ).

The effect in AF-related rotor dynamics of intersubject variability in ionic properties was evaluated by using computer simulations of spherical shell of active tissue consisting of a $3 \mathrm{~cm}$ radius sphere incorporating 163,842 nodes, with an average inter-nodal distance of $283.25 \pm 18.42 \mu \mathrm{m}[4,6]$ (Figure 1B). 

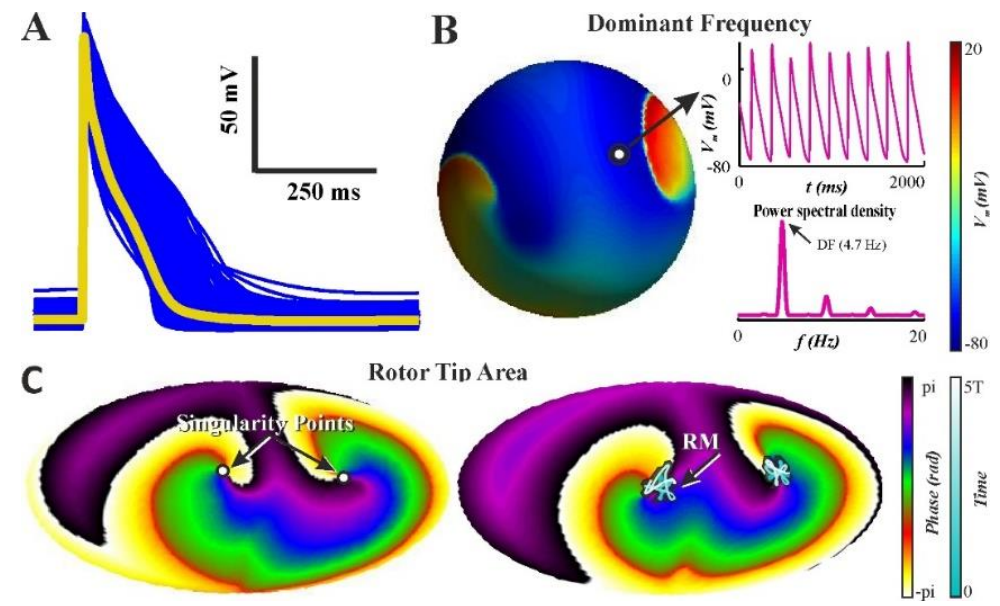

Figure 1. Population of models and re-entry biomarkers (A) Population of Atrial Fibrillation models simulated, original Koivumaki AF model in yellow. (B) Membrane voltage in sphere simulations according to a color scale. Insets show transmembrane voltage and power spectral density for a given node, illustrating the dominant frequency (DF). (C) Phase maps in Aitoff projection of the sphere. Left: detection of the rotor tip or singularity point; right: meandering of the rotor tip according to a color scale.
Reentrant activity was induced in the spherical model by using a cross stimulation protocol in three different scenarios (1) basal conditions, (2) after a 50\% decrease in the $\mathrm{I}_{\mathrm{Na}}$ conductance and (3) after a 50\% decrease in the $\mathrm{I}_{\mathrm{CaL}}$ conductance. Fibrillation activity of models that could maintain a reentrant activity was characterized with two different AF characteristic biomarkers: the dominant frequency (DF) and the area of rotor meandering (RM) (See Figure 1B and C [4]).

The ion channel parameters and biomarkers were compared in order to highlight the mechanisms of $\mathrm{AF}$ termination and the parameters involved. Mann-Whitney U-test, which is robust against non-Gaussian distributions, was used to evaluate statistical significance between variables $(\mathrm{p}<0.01)$.

Finally, a self-organizing map (SOM) analysis was performed in order to evaluate the capacity of this artificial neural network based technology at the time of classifying models based on the ionic parameters introduced.

\section{Results}

As described in Figure 2 arrhythmias were selfterminated in 47/173 (27\%) of the models during the first seconds of simulated reentrant activity. Our previous results [4] show how AF maintenance was associated with stable rotors during the entire simulation while in terminating arrhythmias rotor cores drifted up to collision where, the arrhythmia terminated.

Block of sodium current terminated AF by inducing collisions between rotors in 64/126 models (note 126 models in which the reentrant was not self-terminated in basal conditions), a more detailed description of $\mathrm{AF}$ termination by $\mathrm{I}_{\mathrm{Na}}$ partial block is detailed in Figure 3 . Partial calcium block produced the reentrant termination in $38(30.2 \%)$ models. Effect of sodium and calcium block was exclusive in 52 AF models: 39 models (60.9\%) which terminated by sodium block did not terminate by calcium block, whereas 13 of the AFs which terminated by calcium block did not terminate with sodium block $(34.2 \%)$. It shows how even the mechanism of termination is the same (i.e. rotor core collision) different treatments should be applied to different patients.

Figure 3A shows a comparison of the distribution of each ionic current parameter between the models in which the fibrillation terminated and those in which it was perpetuated.

Sodium, $\mathrm{I}_{\mathrm{Kur}}$ and $\mathrm{I}_{\mathrm{K} 1}$ conductivities ( $\mathrm{g}_{\mathrm{Na}}, \mathrm{g}_{\mathrm{Kur}}$ and $\mathrm{g}_{\mathrm{K} 1}$ ) were the parameters that presented significant differences between both groups. It can be observed how a low availability of $\mathrm{I}_{\mathrm{Na}}$ and $\mathrm{I}_{\mathrm{Kur}}$ and a high $\mathrm{I}_{\mathrm{K} 1}$ conductivity easies reentrant behavior termination due to of $I_{N a}$ partial block.

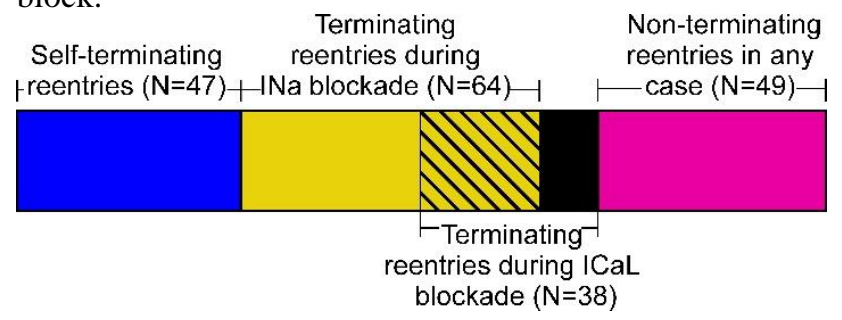

Figure 2. Distribution of the models in the population according with reentrant activity maintenance under different conditions. During basal conditions 126 of the $173(72.8 \%)$ models sustained stable reentries. Partial block of sodium terminated AF by collision between rotors in 64 fibrillation models $(50.8 \%)$ whereas partial calcium block produced the reentrant termination in 38 $(30.2 \%)$. With both treatments, termination was associated with an increase in meandering and collision between rotors. Ef-fect of sodium and calcium block was exclusive in 52 AF models: 39 models (60.9\%) which terminated by sodium block did not terminate by calcium block, whereas 13 of the AFs which terminated by calcium block did not terminate with sodium block (34.2\%). 


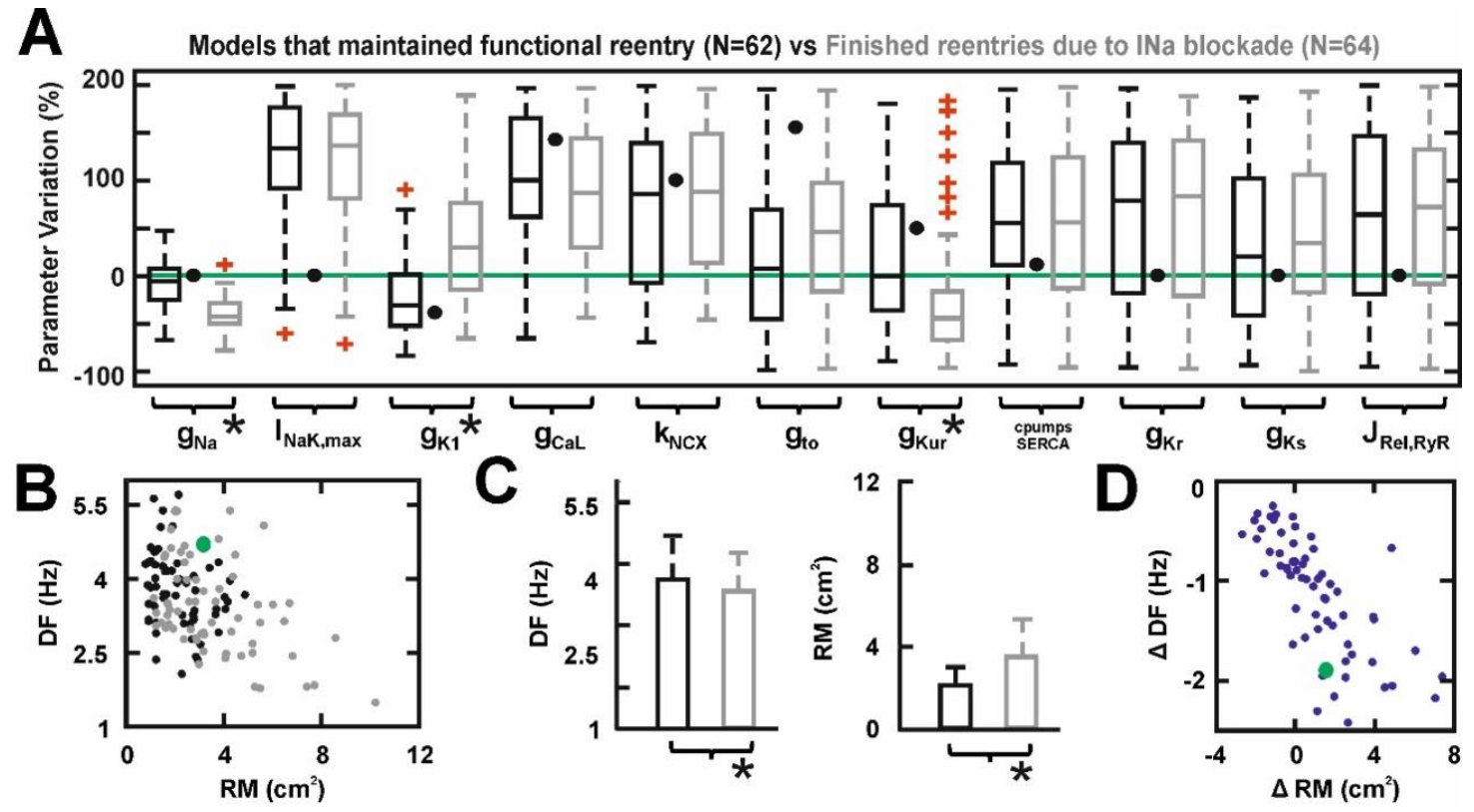

Figure 3. Effect of $I_{N a}$ partial block. Panel A compares the parameters associated to ionic currents of the 62 models which maintained the reentrant activity (black) after $\mathrm{I}_{\mathrm{Na}}$ partial block with those that could not (i.e 64 models in gray). It can be observed how a huge variability in ionic parameters allow physiological APs, tissue models displaying arrhythmia termination were associated with higher conductivity of $\mathrm{I}_{\mathrm{K} 1}\left(\mathrm{~g}_{\mathrm{K} 1}\right)$, and both reduced sodium conductivity $\left(\mathrm{g}_{\mathrm{Na}}\right)$ and fast potassium current ( $\mathrm{g}_{\mathrm{Kur}}$ ) conductivity. Panel B and C show the scattergram and bar graphs of DF and RM prior to $\mathrm{I}_{\mathrm{Na}}$ partial block showing how the mechanism of AF termination was related with higher RM $(3.58 \pm 1, .97 \mathrm{vs} 2 ., 13 \pm 1 ., 04 \mathrm{~cm} 2$, $\mathrm{p}<0 ., 01)$. Panel $\mathrm{C}$ shows how $\mathrm{I}_{\mathrm{Na}}$ partial block resulted in a reduced DF in all models, while RM was increased in most of the models.

It is according with our previous results in which the effect of $\mathrm{I}_{\mathrm{CaL}}$ partial block was evaluated and the specific ratios between $\mathrm{g}_{\mathrm{Na}}$ and $\mathrm{g}_{\mathrm{K} 1}$ were essential in the antiarrhythmic effect of $\mathrm{I}_{\mathrm{CaL}}$ partial block.

Figure $3 \mathrm{~B}$ and $\mathrm{C}$ show the distribution of DF and RM in the models in which $\mathrm{I}_{\mathrm{Na}}$ partial block terminated the arrhythmia and those in which not, it can be observed how the models in which the arrhythmia terminated presented a basal lower DF and more interestingly that AF termination was related with higher RM $(3.58 \pm 1.97$ vs $\left.2.13 \pm 1.04 \mathrm{~cm}^{2}, \mathrm{p}<0.01\right)$. It highlights the mechanism of $\mathrm{AF}$ termination by collision of rotor cores.

Figure 3D shows how $\mathrm{I}_{\mathrm{Na}}$ partial block resulted in a decrease of DF in all the models and an increase of RM in most of the models. It is in accordance to what it was observed in the effect of $\mathrm{I}_{\mathrm{CaL}}$ partial block in which an increase in RM was observed in most of the models, but in that case the effect in DF was divergent because of the refractory period shorten caused by $\mathrm{I}_{\mathrm{CaL}}$ partial block [4].

Figure 4 show the result of SOM analysis. It can be observed how this artificial neural network procedure was able to classify the models according to according to their similarities regarding the variations on ionic parameters (left panel) and thus estimating the probability of success or not of $\mathrm{I}_{\mathrm{Na}}$ block treatment (right panel). The method was able to classify responder (R) and non-responder $(\mathrm{N})$ models with only two spaces in which both a $\mathrm{R}$ and a $\mathrm{N}$ model was placed at the same time (for two very similar models in terms of ionic profile one resulted to be a responder and the other did not). The map classified responder models on the top and non-responders on the bottom with the only exception of five responder models that were placed in two spaces on the bottom (these models, similar to the non-responder models, resulted to be responders because of a specific ionic profile).

This strategy is being evaluated in order to predict the most appropriate treatment for a given patient according with the expression of the different ionic currents and other biomarkers in an in-silico scenario.

\section{Discussion}

A population of 173 mathematical models which mimics the inter-subject variability of $149 \mathrm{AF}$ patients including rate-dependent response has been used to evaluate the role of each ionic current on AF reentry perpetuation mechanisms by means of 3D spherical models.

The role of $\mathrm{I}_{\mathrm{Na}}, \mathrm{I}_{\mathrm{CaL}}, \mathrm{I}_{\mathrm{Kur}}$ and $\mathrm{I}_{\mathrm{K} 1}$ currents on $\mathrm{AF}$ dynamics and termination of reentrant activity by collision of rotor cores resulted to be fundamental and determinant on the most appropriate antiarrhythmic treatment and in the probability of our model to be a responder or not. 

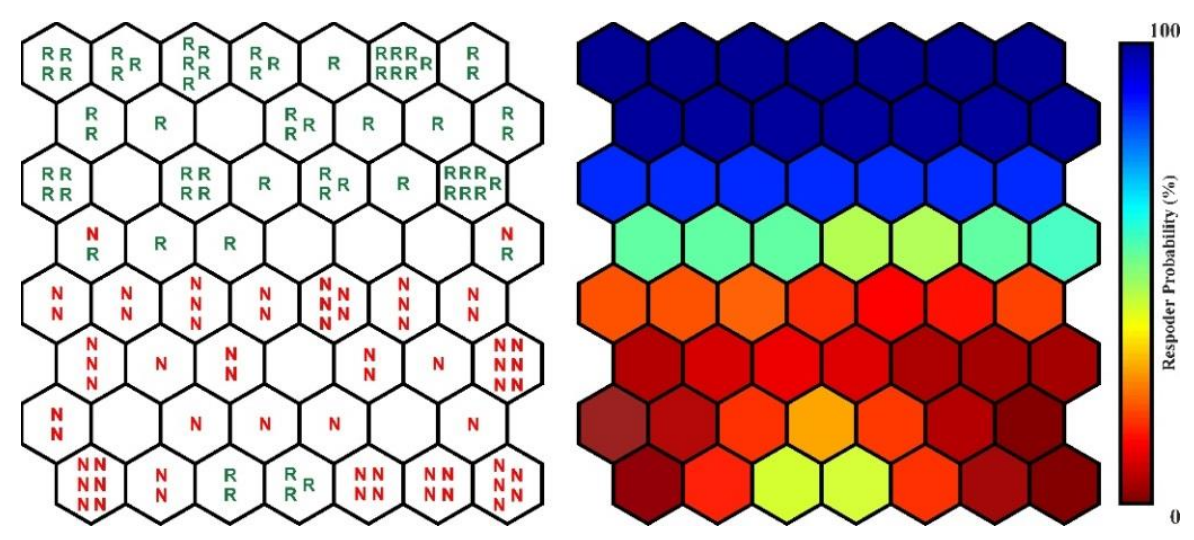

Figure 4. Self-Organizing Map (SOM) analysis for $\mathrm{I}_{\mathrm{Na}}$ partial block. The panel on the left shows the distribution of models that were organized according to their similarities regarding the variations on ionic parameters and the response to the treatment. Panel on the right shows the probability of each specific model to be a responder to sodium channel block treatment according with the distribution of models.

Antiarrhythmic drugs are the main strategy for $\mathrm{AF}$ cardioversion and the maintenance of sinus rhythm, however they are associated with undesired secondary effects and a lack of efficacy[7].

For this reason, the development of new drugs based on mechanistic strategies has attracted the interest of pharmacological companies and research groups $[1,8]$. In this context, the use of tissue simulations is an important tool for the better understanding of rotor dynamics and the mechanisms that brings to AF termination. Our simulations with populations of models have demonstrated that the efficacy of sodium or calcium antiarrhythmic strategy will depend on each patient specific balance between ion currents.

This inter-subject variability approach is a promising tool for the training of classifying techniques based on electrophysiological biomarkers that will bring closer the personalized treatment of cardiac arrhythmias.

\section{Conclusions}

Simulations based on experimentally-calibrated populations of models predict that a significant number of AF patients may require completely different pharmacological strategies depending on their specific electrophysiological properties. Classification techniques based on artificial neural networks are promising at the time of obtaining measurable biomarkers and predict the response probability to different drug treatments.

\section{Acknowledgements}

Supported by: The Spanish Ministry of Economy through the Instituto de Salud Carlos III, FEDER Fondo Europeo de Desarrollo Regional, (PI13/00903, PI13/01882, PI16/01123, DTS16/00160, RIC. RD12.0042.0001); Spanish Ministry of Science and Innovation (IJCI-2014-22178); Spanish Society of Cardiology; Wellcome Trust Fellowship 100246/Z/12/Z.

\section{References}

[1] Pandit SV, Zlochiver S, Filgueiras-Rama D, et al. Targeting atrioventricular differences in ion channel properties for terminating acute atrial fibrillation in pigs. Cardiovasc Res 2011; 89:843-851.

[2] Sanchez C, Bueno-Orovio A, Wettwer E, et al. InterSubject Variability in Human Atrial Action Potential in Sinus Rhythm versus Chronic Atrial Fibrillation. PLoS One 2014; 9:e105897.

[3] Climent AM, Guillem MS, Fuentes L, et al. The Role of Atrial Tissue Remodeling on Rotor Dynamics: An In-Vitro Study. Am J Physiol Heart Circ Physiol 2015: 309: H1964H1973.

[4] Liberos A, Bueno-Orovio A, Rodrigo M, et al. Balance between sodium and calcium currents underlying chronic atrial fibrillation termination: $\mathrm{An}$ in silico intersubject variability study. Heart Rhythm 2016; 13:2358-2365.

[5] Koivumaki JT, Seemann G, Maleckar MM, et al. In Silico Screening of the Key Cellular Remodeling Targets in Chronic Atrial Fibrillation. Plos Comput Biol 2014; 10:e1003620.

[6] Rodrigo M, Guillem MS, Climent AM, et al. Body Surface Localization of Left and Right Atrial High Frequency Rotors in Atrial Fibrillation Patients: A ClinicalComputational Study. Heart Rhythm 2014; 11:1584-1591.

[7] Filgueiras-Rama D, Castrejon S, Calvo C, et al. [Basic mechanisms of the new antiarrhythmic drugs in atrial fibrillation]. Arch Cardiol Mex 2012; 82:139-152.

[8] Kneller J, Kalifa J, Zou RQ, et al. Mechanisms of atrial fibrillation termination by pure sodium channel blockade in an ionically-realistic mathematical model. Circ Res 96:E35-E47.

Address for correspondence.

Alejandro Liberos Mascarell.

Hospital GU Gregorio Marañón

Instituto de Investigación Sanitaria Gregorio Marañón Laboratorio de Órganos y Matrices Bioartificiales

Edificio Materno-Infantil

cl O'Donnell 48, 28009, Madrid, España

alejandroliberos@gmail.com 